\title{
IV Diretrizes Brasileiras de Hipertensão Arterial Grupos de Trabalho
}

\begin{tabular}{|c|c|c|}
\hline \multicolumn{2}{|c|}{$\begin{array}{l}\text { Grupo } 1 \text { - DIAGNÓSTICO E CLASSIFICAÇÃO } \\
\text { Coordenador: Marco Antonio Mota Gomes (AL) }\end{array}$} & \multirow{2}{*}{$\begin{array}{l}\text { José Xavier de Mello Filho (MA) } \\
\text { Luiz Carlos Bodanese (RS) }\end{array}$} \\
\hline Angela Maria Geraldo Pierin (SP) & Ayrton Pires Brandão (RJ) & \\
\hline Antonio Silveira Sbissa (PR) & Cibeli I. Saad Rodrigues (SP) & \\
\hline Armando da Rocha Nogueira (RJ) & Edgar Pessoa de Mello (PE) & Sebastião Ferreira Filho (MG) \\
\hline \multicolumn{3}{|c|}{$\begin{array}{l}\text { Grupo } 2 \text { - INVESTIGAÇÃO CLÍNICO-LABORATORIAL E DECISÃO TERAPÊUTICA } \\
\text { Coordenador: Fernando Nobre (SP) }\end{array}$} \\
\hline Agostinho Tavares (SP) & José Márcio Ribeiro (MG) & Pedro Jabur (SP) \\
\hline Antonio Carlos Lopes (SP) & Luiz Introcaso (DF) & Raimundo Marques Nascimento (MG) \\
\hline Jorge Pinto Ribeiro (RS) & Marcelo Corrêa (RJ) & Roberto de Sá Cunha (ES) \\
\hline José Carlos Aydar Ayoub (SP) & Mario Maranhão (PR) & Rogério Andrade Mulinari (PR) \\
\hline \multicolumn{3}{|c|}{$\begin{array}{l}\text { Grupo } 3 \text { - ABORDAGEM MULTIPROFISSIONAL } \\
\text { Coordenador: Carlos Alberto Machado (SP) }\end{array}$} \\
\hline Adriana Avila (SP) & Maria Cecília G. Marinho Arruda (SP) & Nárcia Elisa B. Kohlmann (SP) \\
\hline Clóvis Oliveira Andrade (SE) & Maria Fátima Azevedo (RN) & Neide de Jesus (BA) \\
\hline João Carlos Rocha (SP) & Maria Helena C. Carvalho (SP) & Paulo César da Veiga Jardim (GO) \\
\hline Margarida Maria Veríssimo Lopes (CE) & Marilda Novaes Lipp (SP) & \\
\hline \multicolumn{3}{|c|}{ Grupo 4 - TRATAMENTO NÃO-MEDICAMENTOSO } \\
\hline Coordenador: Celso Amodeo (SP) & & Fátima Lúcia Machado Braga (PE) \\
\hline Carlos Eduardo Negrão (SP) & Eli Toscano (DF) & Hilton de Castro Chaves Jr. (PE) \\
\hline Celso Ferreira (SP) & Eliuden Galvão de Lima (ES) & Joel Heiman (SP) \\
\hline Cláudio Pereira da Cunha (PR) & Estelamaris Tronco Monego (GO) & Tales de Carvalho (SC) \\
\hline \multicolumn{3}{|c|}{ Grupo 5 - TRATAMENTO MEDICAMENTOSO } \\
\hline Coordenador: Osvaldo Kohlmann Jr. & & Natalino Salgado Filho (MA) \\
\hline Alvaro Avezum (SP) & Gilson Feitosa (BA) & Rafael Leite Luna (RJ) \\
\hline Artur Beltrame Ribeiro (SP) & Harue Ohashi (SP) & Roberto Jorge da Silva Franco (SP) \\
\hline Carlos Alberto Gomes (MG) & José Antonio Franchini Ramirez (SP) & Robson Augusto dos Santos (MG) \\
\hline Dante Marcelo Artigas Giorgi (SP) & Marcelo Marcondes Machado (SP) & Wille Oigman (RJ) \\
\hline \multicolumn{3}{|c|}{$\begin{array}{l}\text { Grupo } 6 \text { - TRATAMENTO DA HIPERTENSÃO ARTERIAL EM SITUAÇÕES ESPECIAIS } \\
\text { Coordenador: Istênio Fernandes Pascoal (DF) }\end{array}$} \\
\hline Airton Massaro (SP) & Ivan Cordovil (RJ) & Maurício Wajngarten (SP) \\
\hline Álvaro Nagib Atallah (SP) & José Egídio Paulo de Oliveira (RJ) & Roberto Dischinger Miranda (SP) \\
\hline Andréa Brandão $(\mathrm{RJ})$ & José Geraldo L. Ramos (RS) & Soubihe Kahhale (SP) \\
\hline Elizabete Viana de Freitas (RJ) & Maria Teresa Zanella (SP) & Vera Koch (SP) \\
\hline \multicolumn{3}{|l|}{ Grupo 7 - PREVENÇÃO PRIMÁRIA } \\
\hline Coordenador: Décio Mion Jr. (SP) & & Marcos Ausenka Ribeiro (SP) \\
\hline Armênio C. Guimarães (BA) & Eduardo B. Coelho (SP) & Michel Batlouni (SP) \\
\hline Catia Sueli Palmeira (BA) & Fernando Antonio Almeida (SP) & Paulo Lotufo (SP) \\
\hline Claudia Lucia de Moraes Forjaz (SP) & Isabel Cristina Estefano Pellizari (SP) & Regina Teresa Capelari (SP) \\
\hline \multicolumn{3}{|c|}{$\begin{array}{l}\text { Grupo } 8 \text { - EPIDEMIOLOGIA } \\
\text { Coordenador: Lucélia C. Magalhães (BA) }\end{array}$} \\
\hline Abrahão Afiune Neto (GO) & Ana Luisa de Souza (GO) & Marcus V. Bolívar Malachias (MG) \\
\hline Abrão Cury (SP) & Flavio Danni Fuchs (RS) & Romero Bezerra (DF) \\
\hline Alci Moreira (MG) & Ines Lessa (BA) & Sandra Fuchs (RS) \\
\hline \multicolumn{3}{|c|}{$\begin{array}{l}\text { Grupo } 9 \text { - HIPERTENSÃO SECUNDÁRIA } \\
\text { Coordenador: José Nery Praxedes (SP) }\end{array}$} \\
\hline Antonio Cambara (SP) & Helio B. Silva (SP) & Luiz Bortolotto (SP) \\
\hline Antonio Marmo Lucon (SP) & João Egidio Romão Jr. (SP) & Luis Celso Matavelli (SP) \\
\hline Berenice Mendonça (SP) & José Gastão Rocha Carvalho (PR) & Maria Eliete Pinheiro (SP) \\
\hline Flavio Borelli (SP) & José Luiz Santello (SP) & Valéria Guimarães (DF) \\
\hline
\end{tabular}

\section{Grau de Recomendação}

Em sintonia com a tendência científica mundial e a orientação da Associação Médica Brasileira, as IV Diretrizes Brasileiras de Hipertensão fundamentam suas orientações segundo Graus de Recomendação baseados em níveis de evidência dos estudos clínicos de referência:

Grau A - grandes ensaios clínicos aleatorizados e metanálises.

Grau B -estudos clínicos e observacionais bem desenhados.

Grau C-relatos e séries de casos.

Grau D - publicações baseadas em consensos e opiniões de especialistas. 


\section{Brazilian Guidelines on Arterial Hypertension Work Groups}

\begin{tabular}{|c|c|c|}
\hline \multicolumn{3}{|c|}{ Group 1 - DIAGNOSIS AND CLASSIFICATION } \\
\hline Angela Maria Geraldo Pierin (SP) & Ayrton Pires Brandão (RJ) & $\begin{array}{l}\text { José Xavier de Mello Filho (MA) } \\
\text { Luiz Carlos Bodanese (RS) }\end{array}$ \\
\hline Antonio Silveira Sbissa (PR) & Cibeli Isaac Saad Rodrigues (SP) & Paulo Toscano (PA) \\
\hline Armando da Rocha Nogueira (RJ) & Edgar Pessoa de Mello (PE) & Sebastião Ferreira Filho (MG) \\
\hline \multicolumn{3}{|c|}{$\begin{array}{l}\text { Group } 2 \text { - CLINICAL AND LABORATORY INVESTIGATION and THERAPEUTIC DECISION } \\
\text { Coordinator: Fernando Nobre (SP) }\end{array}$} \\
\hline Agostinho Tavares (SP) & José Márcio Ribeiro (MG) & Pedro Jabur (SP) \\
\hline Antonio Carlos Lopes (SP) & Luiz Introcaso (DF) & Raimundo Marques Nascimento (MG) \\
\hline Jorge Pinto Ribeiro (RS) & Marcelo Corrêa (RJ) & Roberto de Sá Cunha (PR) \\
\hline José Carlos Aydar Ayoub (SP) & Mario Maranhão (PR) & Rogério Andrade Mulinari (PR) \\
\hline \multicolumn{3}{|c|}{$\begin{array}{l}\text { Group } 3 \text { - MULTIPROFESSIONAL APPROACH } \\
\text { Coordinator: Carlos Alberto Machado (SP) }\end{array}$} \\
\hline Adriana Avila (SP) & Maria Cecília G. Marinho Arruda (SP) & Nárcia Elisa B. Kohlmann (SP) \\
\hline Clóvis Oliveira Andrade (SE) & Maria Fátima Azevedo (RN) & Neide de Jesus (BA) \\
\hline João Carlos Rocha (SP) & Maria Helena C. Carvalho (SP) & Paulo César da Veiga Jardim (GO) \\
\hline Margarida Maria Veríssimo Lopes (CE) & Marilda Lipp (SP) & \\
\hline \multicolumn{3}{|l|}{ Group 4 - NON-DRUG TREATMENT } \\
\hline Coordinator: Celso Amodeo (SP) & & Fátima Lúcia Machado Braga (PE) \\
\hline Carlos Eduardo Negrão (SP) & Eli Toscano (DF) & Hilton de Castro Chaves Jr. (PE) \\
\hline Celso Ferreira(SP) & Eliuden Galvão de Lima (ES) & Joel Heiman (SP) \\
\hline Cláudio Pereira da Cunha (PR) & Estelamaris Tronco Monego (GO) & Tales de Carvalho (SC) \\
\hline \multirow{2}{*}{\multicolumn{3}{|c|}{$\begin{array}{l}\text { Group } 5 \text { - DRUG TREATMENT } \\
\text { Coordinator: Osvaldo Kohlmann Jr. (SP) }\end{array}$}} \\
\hline & & Natalino Salgado Filho (MA) \\
\hline Alvaro Avezum (SP) & Gilson Feitosa (BA) & Rafael Leite Luna (RJ) \\
\hline Artur Beltrame Ribeiro (SP) & Harue Ohashi (SP) & Roberto Jorge da Silva Franco (SP) \\
\hline Carlos Alberto Gomes (MG) & José Antonio Franchini Ramirez (SP) & Robson Augusto dos Santos (MG) \\
\hline Dante Marcelo Artigas Giorgi (SP) & Marcelo Marcondes Machado (SP) & Wille Oigman (RJ) \\
\hline \multicolumn{3}{|c|}{$\begin{array}{l}\text { Group } 6 \text { - TREATMENT OF ARTERIAL HYPERTENSION IN SPECIAL SITUATIONS } \\
\text { Coordinator: Istênio Fernandes Pascoal (DF) }\end{array}$} \\
\hline Airton Massaro (SP) & Ivan Cordovil (RJ) & Maurício Wajngarten (SP) \\
\hline Álvaro Nagib Atallah (SP) & José Egídio Paulo de Oliveira (RJ) & Roberto Dischinger Miranda (SP) \\
\hline Andréa Brandão (RJ) & José Geraldo L. Ramos (RS) & Soubihe Kahhale (SP) \\
\hline Elizabete Viana de Freitas (RJ) & Maria Teresa Zanella (SP) & Vera Koch (SP) \\
\hline \multicolumn{3}{|l|}{ Group 7 - PRIMARY PREVENTION } \\
\hline Coordinator: Décio Mion Jr. (SP) & & Marcos Ausenka Ribeiro (SP) \\
\hline Armênio C. Guimarães (BA) & Eduardo B. Coelho (SP) & Michel Batlouni (SP) \\
\hline Catia Sueli Palmeira (BA) & Fernando Antonio Almeida (SP) & Paulo Lotufo (SP) \\
\hline Claudia Lucia de Moraes Forjaz (SP) & Isabel Cristina Estefano Pellizari (SP) & Regina Teresa Capelari (SP) \\
\hline \multicolumn{3}{|l|}{$\begin{array}{l}\text { Group } 8 \text { - EPIDEMIOLOGY } \\
\text { Coordinator: Lucélia C. Magalhães (BA) }\end{array}$} \\
\hline Abrahão Afiune Neto (GO) & Ana Luisa de Souza (GO) & Marcus V. Bolívar Malachias (MG) \\
\hline Abrão Cury (SP) & Flavio Danni Fuchs (RS) & Romero Bezerra (DF) \\
\hline Alci Moreira (MG) & Inês Lessa (BA) & Sandra Fuchs (RS) \\
\hline \multicolumn{3}{|c|}{$\begin{array}{l}\text { Group } 9 \text { - SECONDARY HYPERTENSION } \\
\text { Coordinator: José Nery Praxedes (SP) }\end{array}$} \\
\hline Antonio Cambara (SP) & Helio B. Silva (SP) & Luiz Bortolotto (SP) \\
\hline Antonio Marmo Lucon (SP) & João Egidio Romão Jr. (SP) & Luis Celso Matavelli (SP) \\
\hline Berenice Mendonça (SP) & José Gastão Rocha Carvalho (PR) & Maria Eliete Pinheiro (SP) \\
\hline Flavio Borelli (SP) & José Luiz Santello (SP) & Valéria Guimarães (DF) \\
\hline
\end{tabular}

\section{Grade of Recommendation}

In accordance with the global scientific trend and the guidelines of the Brazilian Medical Association, the IV Brazilian Guidelines on Hypertension (IV Diretrizes Brasileiras de Hipertensão) set their guidelines according to Grades of Recommendation based on the levels of evidence from clinical reference studies:

Grade A - major randomized clinical assays and meta-analyses.

Grade B - well designed clinical and observational assays.

Grade $\mathbf{C}$-reports and case series.

Grade D - publications based on consensus and specialized opinions. 